\title{
Imaging of Neuromodulation and Surgical Interventions for Epilepsy
}

\author{
(D) M.E. Adin, (DD.D. Spencer, (DE. Damisah, (D)A. Herlopian, (D).L. Gerrard, and (D)R.A. Bronen
}

\begin{abstract}
SUMMARY: About one-third of epilepsy cases are refractory to medical therapy. During the past decades, the availability of surgical epilepsy interventions has substantially increased as therapeutic options for this group of patients. A wide range of surgical interventions and electrophysiologic neuromodulation techniques are available, including lesional resection, lobar resection, thermoablation, disconnection, multiple subpial transections, vagus nerve stimulation, responsive neurostimulation, and deep brain stimulation. The indications and imaging features of potential complications of the newer surgical interventions may not be widely appreciated, particularly if practitioners are not associated with comprehensive epilepsy centers. In this article, we review a wide range of invasive epilepsy treatment modalities with a particular focus on their postoperative imaging findings and complications. A state-of-the-art treatment algorithm provides context for imaging findings by helping the reader understand how a particular invasive treatment decision is made.
\end{abstract}

ABBREVIATIONS: ANT = anterior thalamic nucleus; ATL = anterior temporal lobectomy; DBS = deep brain stimulation; EEG $=$ electroencephalogram; FGATIR = fast gray matter acquisition T1 inversion recovery; LITT = laser interstitial thermal therapy; MST = multiple subpial transection; MTS = mesial temporal sclerosis; RNS = responsive neurostimulation; VNS = vagus nerve stimulation

E pilepsy, a recurrent spontaneous seizure disorder, is the third leading cause of neurologic symptoms, with a prevalence of approximately $1 \%$. In about one-third of cases, optimal seizure control cannot be achieved with medications alone, leading to significant morbidity and mortality. Surgical treatment for epilepsy was pioneered by Dr Victor Horsley in 1886 , by resecting a focus of traumatic scar tissue and the surrounding tissue in a 22 -year-old patient. ${ }^{1}$ Currently, resective surgery is the technique that is most likely to result in seizure freedom for patients with pharmacoresistant epilepsy. ${ }^{2}$ Complete resection of a focal MR imaging abnormality causing medically intractable epilepsy may result in $>1$ year of seizure freedom in a substantial proportion of patients.

Surgical interventions for refractory epilepsy include thermoablation, functional hemispherectomy, corpus callosotomy, and cortical transection as well as resection. During the past

Received March 9, 2021; accepted after revision April 30.

From the Departments of Radiology and Biomedical Imaging (M.E.A., R.A.B.), Neurosurgery (D.D.S., E.D., J.L.G.), and Neurology (A.H.), Yale School of Medicine, New Haven, Connecticut.

Paper previously presented as an educational exhibit at: Annual Meeting of the American Society of Neuroradiology, May 22-26, 2021; Virtual.

Please address correspondence to Mehmet Emin Adin, MD, 20 York St, New Haven, CT, 06510; e-mail: Emin.adin@gmail.com; @emin_adin

- Indicates open access to non-subscribers at www.ajnr.org

Indicates article with online supplemental data.

http://dx.doi.org/10.3174/ajnr.A7222 decade, the availability of epilepsy interventions has increased substantially beyond the traditional resective surgical treatments. These modalities include 3 FDA-approved neuromodulation devices. The specific type of surgical intervention is determined for each patient after extensive investigation, which sometimes includes localization via intracranial electrodes (Table and Fig 1).

The indications, imaging findings, and imaging features of potential complications of the newer interventions may not be

\section{Types of surgical and neuromodulation epilepsy interventions}

\begin{tabular}{l} 
Interventions \\
\hline Resective surgery \\
Temporal lobe \\
Temporal lobectomy (characteristically for MTS) \\
Lesionectomy \\
Extratemporal lobe resections \\
Hemispherectomy \\
Functional hemispherectomy \\
Hemispherotomy, disconnection \\
Nonresective surgery \\
Callosotomy, partial vs complete \\
Multiple subpial transection \\
Laser ablation surgery \\
Radiosurgery \\
Neuromodulation \\
Vagus nerve stimulation \\
Responsive neurostimulation \\
Deep brain stimulation \\
\hline
\end{tabular}




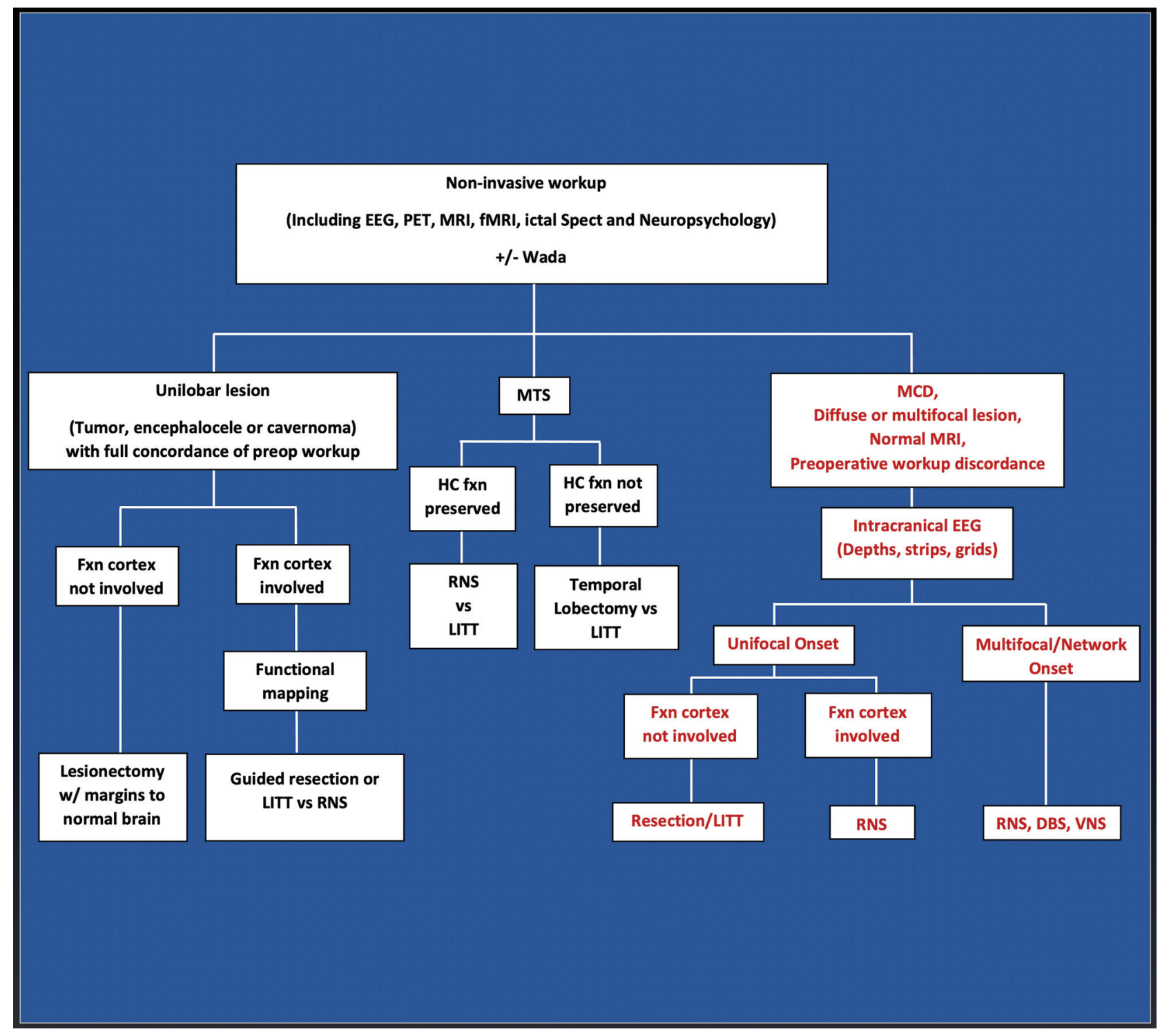

FIG 1. Intractable epilepsy decision tree for surgical intervention. Noninvasive investigations indicate whether a patient is a candidate for surgical resection of unilobar lesions or MTS, or whether an intracranial EEG study is needed for further localization (ie, MCD, diffuse or multifocal lesions, normal MR imaging findings, or discordant investigations). For those with unilobar lesions, surgical treatment is based on whether the functional cortex (defined as verbal memory, sensorimotor cortex, and language regions) is involved, determined by functional electrical stimulation mapping. RNS and possibly LITT are used to treat MTS in those with preserved hippocampal function, as opposed to temporal lobectomy for those with poor hippocampal memory. Nonfocal multifocal or network-onset seizures are treated by RNS, DBS, or VNS. Fxn cortex indicates functional cortex; HC fxn, hippocampal function; MCD, malformation of cortical development; Wada, intracarotid amobarbital testing.

widely appreciated, particularly if practitioners are not associated with comprehensive epilepsy centers. In this article, we review a wide range of invasive epilepsy treatment modalities including electrophysiologic neuromodulation, with a particular focus on their postoperative imaging findings and complications. We will initially explore preoperative imaging aspects of intracranial electrodes, then discuss resective and nonresective surgeries, and conclude with neuromodulation. The focus will be on imaging findings and complications specifically associated with epilepsy surgery.

\section{Overview of Surgical Intervention}

The goal of resective surgery is to render the patient seizure-free by optimizing the resection of epileptogenic brain and avoiding neurologic and cognitive sequelae. Identification of the epileptogenic foci is required for all types of interventions for pharmacoresistant epilepsy and is determined initially through noninvasive means, including history and physical examination, audiovisual electroencephalogram (EEG) monitoring, neuropsychologic testing, MR imaging, PET, and sometimes SPECT and magnetoencephalography. Task-based fMRI is widely used for identification of eloquent cortices (sensory-motor and language) before resective surgery and has largely replaced Wada, an invasive test used for determination of the dominant hemisphere for language activity (see the algorithm in Fig 1). In a variety of cases, an intracranial EEG investigation may be necessary. During approximately the past 2 decades, evidence of a new concept of epilepsy has evolved, 

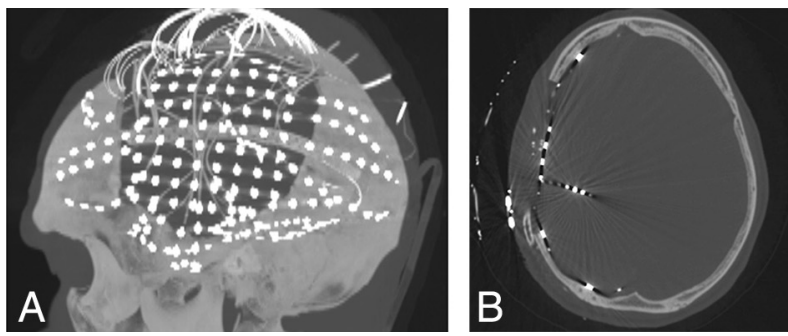

FIG 2. Postelectrode placement with sagittal MIP $(A)$ from axial CT images $(B)$ demonstrating a subdural electrode grid over an eloquent region (eg, visual-spatial region) and subdural strip and depth electrodes. To determine the exact localization of right temporoparietal seizure onset, we coregistered CT scans to MR images (C) because electrode positions are better identified on CT. A depth electrode is placed into the subependymal heterotopia to assess whether seizures are originating there (arrow).
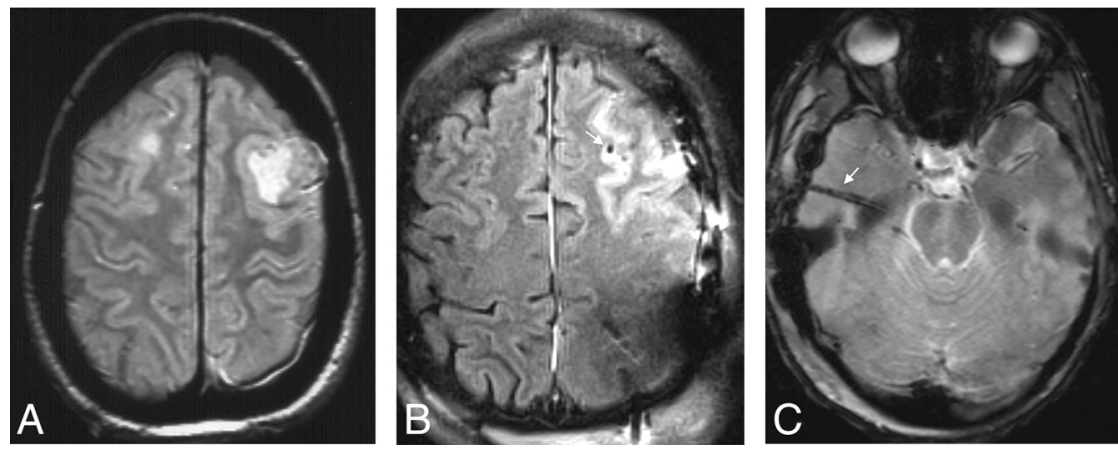

FIG 3. Electrode complications in 2 patients include edema $(A)$ and subarachnoid hemorrhage $(B)$, possibly related to vascular injury on FLAIR axial images (arrow points to adjacent electrode). Imaging findings of the electrode tracts (arrow) include hemosiderin on gradient recalled-echo imaging $(C)$ and contrast enhancement on axial TTWI (not shown here).

with major implications for treatment; this theory suggests that epilepsy is a disorder of the neural networks in both generalized and focal epilepsies. ${ }^{3}$ As a network disorder, destruction (through resection or other surgical techniques) or disruption (through neurostimulation of an EEG focus or the thalamus) of critical nodes or hubs in the network can lead to improvement in seizure outcomes. Convergence of localization of brain dysfunction through seizure semiology, EEG, and neuroimaging helps to determine the epileptogenic foci or critical hub in the network.

A variety of short- and long-term complications is associated with specific types of interventions for epilepsy. Infection and hemorrhage can be seen in all types of interventional epilepsy treatments, though they are rarely encountered. Fever, headache, unexpected seizures, and lethargy as well as other clinical findings of meningoencephalitis may raise suspicion of infectious complications. On imaging, the surgical route and instrumentations must be scrutinized for signs of infection, including, but not limited to, parenchymal enhancement, fluid collection, and restricted diffusion. Interval changes on follow-up imaging may help identify infection, though it may not be possible to reach a conclusion based on imaging alone, particularly in the early postsurgical period due to misleading expected findings, as discussed later in the respective sections.

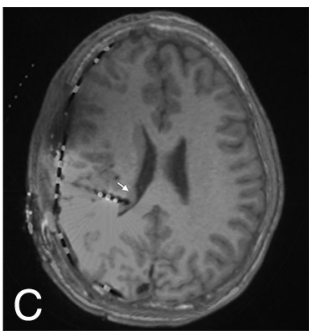

\section{Intracranial Electrodes}

Intracranial electrodes are used when noninvasive investigations fail to localize the ictal onset or when the workup reveals findings discordant with an MR imaging lesion, occurring in 25\% of patients with pharmacoresistant epilepsy. The need for intracranial electrodes is often necessary in cases without an MR imaging-identifiable focal lesion (focal lesion such as cavernoma, temporal lobe encephalocele, or tumor). At our institution, we investigate diffuse pathologic changes (such as large ischemic or inflammatory changes) and malformations of cortical development with intracranial electrodes because the seizure foci may not be directly linked to the abnormality on MR imaging or may be more widespread. Because focal cortical dysplasia, which now accounts for a substantial proportion of pharmacoresistant epilepsy, is often difficult to detect by MR imaging and the seizure focus may be divergent, intracranial electrodes are required. Intracranial electrodes may also be used to determine the relationship between nondispensable cortex (e.g., language or motor areas) and an epileptogenic focus. Electrodes are made of MR imaging-compatible materials such as platinum-iridium and can be imaged safely on a $1.5 \mathrm{~T}$ scanner. ${ }^{4}$ Despite the lack of American Society for Testing and Materials (ASTM) MR imaging labeling for some commercially available brands of platinum-iridium-based intracranial electrodes (because they were on the market long before 2005, when the FDA manual was released), there have been decades of excellent MR imaging safety records using transmit-receive head coils.

Electrodes can be placed using frame-based or frameless stereotactic guidance, or with robotic assistance. There are 2 main types of intracranial electrodes: 1) subdural grid or strip electrodes for neocortical coverage, and 2) depth electrodes that extend into the parenchyma (Fig 2, and Online Supplemental Data).

Precise identification of the intracranial electrodes is crucial because they play a central role in diagnosis and treatment. CT and MR images obtained after electrode placement allow coregistration of the exact location of electrodes defined by CT to MR imaging anatomy and abnormal findings. CT is frequently used early after electrode placement to exclude major intracranial complications, such as hemorrhage, edema, or mass effect. Electrode complications are rare (Fig 3). Punctate hyperintense signal changes on long TR images representing gliosis have been found in $41 \%$ of depth electrode tracks after removal, while hemosiderin, hematoma, enhancement, or calcification from the tracks are rarely seen., 

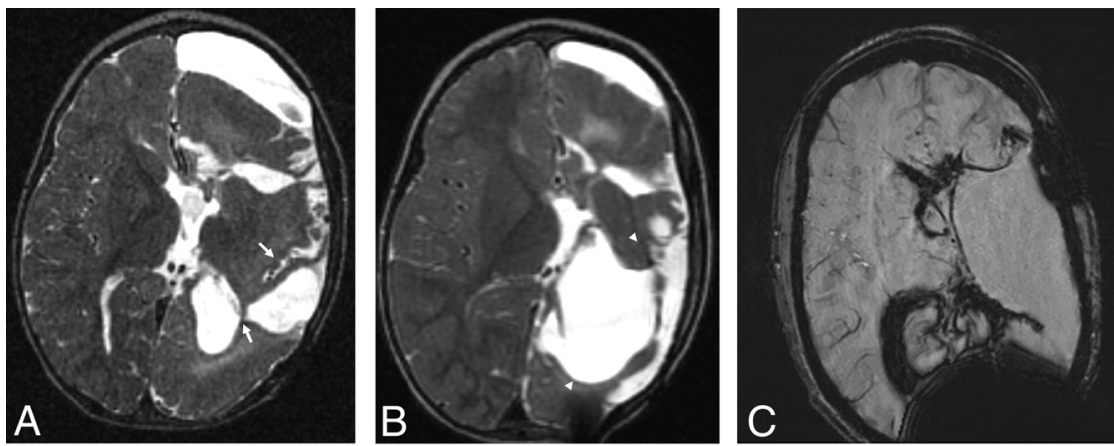

FIG 4. Staged functional hemispherectomy. Initial functional hemispherectomy for hemimegalencephaly consisted of left frontal, parietal, and temporal lobe resections and disconnection of most of the rest of the left hemisphere from the contralateral hemisphere and deep gray matter $(A)$. Persistent seizures from the occipital lobe and insula indicated incomplete disconnection (arrows in $A$, axial T2WI) necessitating further resection of these structures (arrowheads in B, axial T2WI). Hemorrhage and hemosiderosis are complications of larger resections, as seen on axial SWI (C).
It is important to be aware of imaging findings after ATL epilepsy surgery. MR imaging findings may be misleading when performed in the immediate postoperative period, and MR imaging is only indicated when there is high clinical suspicion for potential complications or a necessity for baseline postoperative imaging (such as in tumors). On MR imaging, enhancement of the surgical margin is thin linear within the first 1-5 days but becomes thick linear or nodular (mimicking a tumor appearance) during the first week to month and then disappears (Online Supplemental Data). ${ }^{12}$ Restricted diffusion in the parenchymal rim of ATL resections may occur in half of those imaged within 24 hours

\section{Resective Surgery}

The efficacy and safety of surgery for pharmacoresistant epilepsy have been established by clinical trials in adults ${ }^{7}$ and children. ${ }^{8}$ Surgical decision-making may be relatively straightforward when there is a focal lesion or evidence of mesial temporal sclerosis (MTS) that is proved to cause seizure onset. The most common form of surgical resection is anterior temporal lobectomy (ATL), which is the primary surgical intervention method in refractory MTS. Other temporal lobe resective surgeries include lesionectomy or cortical resection. For extratemporal resections, achieving an excellent outcome is less likely in cases with negative findings on MR imaging, ${ }^{9}$ underscoring the importance of presurgical lesion identification with meticulous imaging.

\section{Temporal Lobectomy}

The standard surgical resection in ATL involves removing the amygdala, hippocampus, and uncus of the parahippocampus, often en bloc, leaving the pia intact. ${ }^{10} \mathrm{~A}$ number of studies have suggested a relationship between the amount of medial temporal lobe tissue resected and good postoperative seizure outcome. Specifically, if imaging reveals residual tissue involved with postoperative seizures, further surgery may result in an additional cure in $50 \%$ of patients. ${ }^{10}$ There are other reasons for surgical failures, including extratemporal seizures mimicking temporal lobe epilepsy, such as from the insula.

In ATL surgery, the cerebral peduncle, posterior cerebral and communicating arteries, the anterior choroidal artery, thalamus, oculomotor nerve, and optic radiation/Meyer loop are at risk of injury. With the advent of microscopic neurosurgery, the incidence of surgical complications has notably decreased, though it is still reported to reach 7\%. ${ }^{11}$ Psychiatric and cognitive disorders, visual field deficits, hemiparesis, language disorders, hemorrhages, infections, and third and fourth nerve dysfunctions are among the clinical complications of ATL surgery. Hemorrhage, usually without mass effect, after ATL may occasionally be seen remote from the surgical site in the cerebellar vermis and folia, thought to be related to CSF loss. $^{10}$ postoperatively, while more extensive involvement may indicate vascular injury. ${ }^{13}$ Extraparenchymal findings include dural enhancement, which may last years, extra-axial fluid lasting 12 months, and pneumocephalus lasting 5 days. ${ }^{12}$ Enlargement, sagging, and increased enhancement in the ipsilateral choroid plexus after ATL should not be mistaken for tumor (Online Supplemental Data). ${ }^{14}$ Ex vacuo enlargement of the ipsilateral temporal horn is almost unequivocally present.

Extratemporal Lobe Resections. Frontal lobe resections are the most common extratemporal surgeries $(48 \%) .{ }^{15}$ In addition to frontal, parietal, and occipital locations (posterior quadrant surgery), the insular, cingulate, and hypothalamic regions are not infrequent extratemporal sites for epilepsy surgery. Extratemporal resective surgery is associated with a higher degree of complicachance of disease cure.

\section{Functional Hemispherectomy/Hemispherotomy}

The hemispherectomy technique is used for patients with multilobar intractable epilepsy, single hemispheric dysfunction such as dysplastic hemimegalencephaly, Rasmussen encephalitis, SturgeWeber syndrome, or ischemic infantile hemiplegic syndrome. However, anatomic hemispherectomy has been linked to lateonset hydrocephalus and superficial hemosiderosis, lethal complications that often occur a decade later. These complications have led to several modifications known as functional hemispherectomy and hemispherotomy (including peri-insular, parasagittal, and endoscopic hemispherotomies), with more limited resection of tissue and greater emphasis on disconnection of the epileptogenic hemisphere and an ensuing decrease in complications (Fig 4). Although intermittent intracranial hemorrhage with resultant hydrocephalus or subsequent siderosis has decreased, these complications still occur, particularly with large-volume reductions associated with functional hemispherectomy, which is more commonly performed with hemimegaloencephaly. In addition to assessing postoperative imaging studies for these complications (which may occur years later), it is critical to confirm that the tions compared with temporal lobe resection, despite a lower 

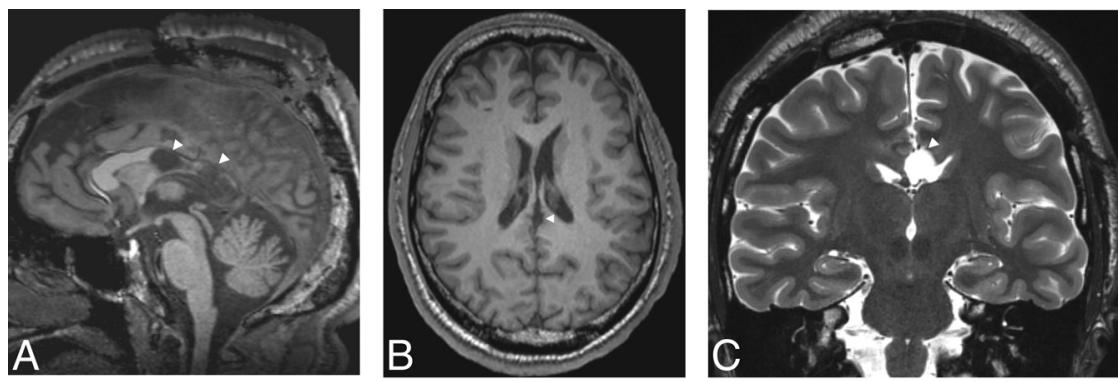

FIG 5. Corpus callosotomy. Disconnection of cerebral hemispheres posteriorly by posterior corpus callosotomy (arrowheads) is shown on sagittal (A) and axial (B) TIWI and coronal T2WI (C). Sagittal images may be inadequate for confirming complete disconnection if the plane of the resection undulates.

hemisphere is disconnected on initial postsurgery MR images, particularly for hemimegalencephaly (Fig 4). The most common cause for failure and reoperation is incomplete disconnection.

\section{Nonresective Surgical Techniques}

Corpus Callosotomy Disconnection Surgery. Corpus callosotomy is a palliative procedure, particularly for intractable drop seizures. By disconnecting the hemispheres, callosotomy disrupts the propagation of seizures rather than cure them. Total or anterior corpus callosotomy are the most common procedure types. The former has a higher likelihood of seizure reduction, while the latter is less likely to result in disconnection syndromes. ${ }^{16}$ Postoperative imaging is often helpful in documenting the extent of callosotomy, particularly when incomplete callosotomy fails to control seizures effectively (Fig 5). In addition to traditional transectional surgery, corpus callosotomy can be accomplished through the use of laser ablative techniques. DTI may be used to confirm whether the intended disconnection has succeeded or to help in planning further interventions against residual connectivity. ${ }^{17}$

Multiple Subpial Transection. Multiple subpial transection (MST) is a surgical intervention that is reserved for patients with medically refractory seizures with epileptogenic foci that overlap the functional (language or motor/sensory) cortex and are therefore not amenable to resection. However, since the approval of neuromodulatory devices such as responsive neurostimulation (RNS), MST is rarely indicated. MST involves severing horizontally oriented cortical fibers of $>5 \mathrm{~mm}$ to disrupt the putative epileptic discharge, while preserving vertically oriented fibers, perpendicular blood vessels, as well as functional activity. ${ }^{18}$ For example, MST for a precentral gyrus epileptogenic site would ideally diminish cortical seizure spread while preserving corticospinal fiber function. Taking advantage of the columnar architecture of the cerebral cortex, the surgical technique is performed as a cluster of small radial cuts, applied through a small pial puncture at the entry point. MST is usually performed as an adjunct to adjacent resective surgery outside the confines of indispensable cortex, though in one-quarter of cases MST has been performed as a stand-alone treatment. ${ }^{10}$ By applying MST to functional brain surrounding the resection of most of an epileptogenic region, the surgeon can minimize the amount of excised cortex and avoid neurologic loss.
MR imaging findings depend on the timing of imaging from the intervention, with initial findings of acute hemorrhagic changes, early vasogenic edema, and fluid pocket formation at the transected cortex and varying degrees of gyral atrophy at later stages (Online Supplemental Data). In their retrospective study of 10 cases, Finet et $\mathrm{al}^{19}{ }^{19}$ reported that $\mathrm{T} 2$-weighted images are the most sensitive sequence in the imaging of MST findings, with no gyral atrophy or gliosis, while Smith $^{20}$ reported cystic changes and gyral atrophy. Cortical atrophy is presumably the result of vascular injury during the procedure, which depends on the extent and depth of the procedure. Signal changes follow the typical course of traumatic brain injury: an initial hemorrhagic area (hyperintense on T1WI), followed by early vasogenic edema (hyperintense on T2WI) and small pockets of fluid signals mid- to long-term (hyperintense on T2WI) and, eventually, varying degrees of gyral atrophy and gliosis. Blood-sensitive MR imaging sequences like SWI and gradient recalled-echo imaging can precisely delineate transection lines due to hemorrhage and hemosiderin, which can persist for years. On imaging, patients with findings of MST are most likely to have other concomitant findings as a result of presurgical intracranial electrode studies and/or adjacent resective surgery.

Laser Ablative Surgery. Laser interstitial thermal therapy (LITT) is an emerging minimally invasive surgical intervention for medically intractable epilepsy, demonstrating satisfactory efficacy for both medial temporal lobe structures and lesions. LITT effectiveness for seizures from MTS is comparable with that of conventional ATL for the short term. ${ }^{21}$ Cavernous malformation, heterotopia, hypothalamic hamartoma, and corpus callosotomy are the more recent applications of LITT in epilepsy treatment. LITT is particularly important for treatment of epileptogenic lesions or abnormalities in the dominant hemisphere, to avoid resective surgery and preserve language. There may be critical clinical implications associated with the specific structures that are targeted; therefore, it is imperative that the location and volume of ablation be detailed in the operative and postoperative imaging reports. ${ }^{21}$

Characteristic time-dependent imaging findings are associated with LITT on MR imaging. ${ }^{22}$ During the early periods (first 2 weeks to 3 months) after the ablative procedure, LITT has a typical target-like appearance with 4 concentric zones, including a peripheral rim of enhancement (Fig 6).

Catheter: In the bull's eye of the ablation region, the laser catheter is imaged as a focal signal void on all sequences. With removal of the catheter, CSF fills the catheter track.

Central zone: T1 hyperintensity and T2 hypointensity from coagulative necrosis are classically associated with methemoglobin, high protein, and engorged blood vessels. Heat likely accelerates conversion of hemoglobin to methemoglobin, which leaks from damaged erythrocytes. ${ }^{22}$ 

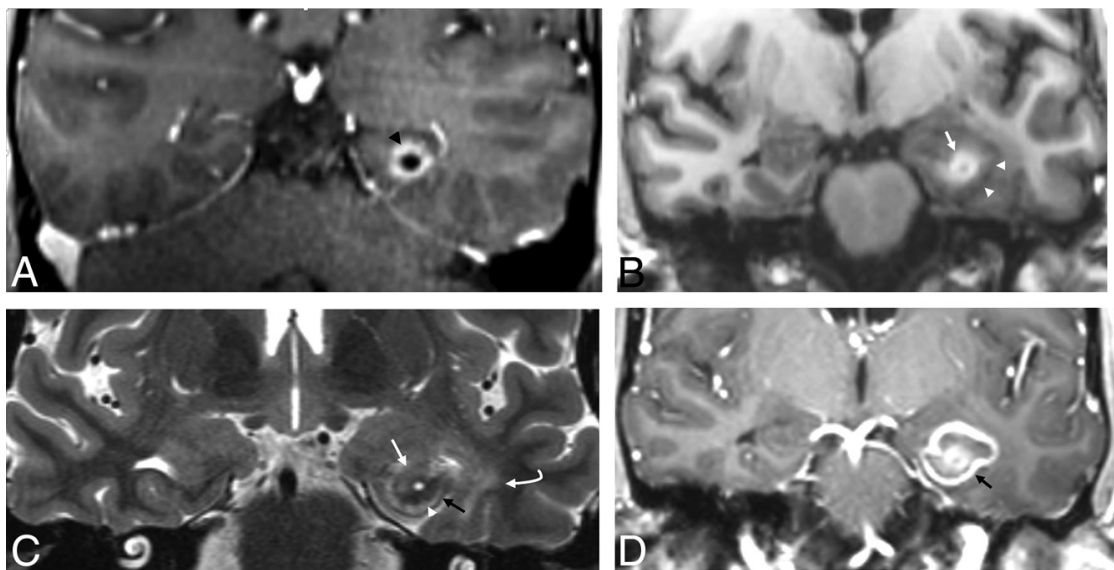

FIG 6. LITT, coronal imaging. Left hippocampal LITT performed in a patient with intractable epilepsy arising from left MTS because of left language dominance. Immediate post-LITT contrast MR imaging $(A)$ shows a catheter (as a central black dot) with surrounding enhancement (black arrowhead) on postcontrast TIW1. The ablated region has enlarged 3 weeks later $(B-D)$. The central zone of coagulative necrosis (white arrow) is hyperintense on precontrast TIWI (B) and hypointense on T2WI (C) signal. These are surrounded by a peripheral zone of necrotizing edema (white arrowhead), which is hypointense on TIWI and hyperintense on T2WI. The peripheral zone is delineated by a rim (black arrow) of signal void on T2WI that enhances on postcontrast imaging $(D)$, defining the ablated area. Vasogenic edema (curved white arrow) can be seen around the ablated region (hyperintense on T2WI and hypointense on precontrast TIWI). Note the focal central CSF intensity signal on $B-D$ from fluid in the laser catheter track.

the lesion can be heterogeneous. However, a T1-hyperintense central zone, an enhancing T2 hypointense peripheral ring, and perilesional white matter edema will always be present.

Radiosurgery. The role, efficacy, and safety of radiosurgery in epilepsy treatment is not well-understood, and its application in temporal lobe epilepsy remains controversial. The antiepileptic effects of radiosurgery were initially observed with treatments of cavernomas and AVMs, in which the primary treatment end point was lesion size reduction to prevent hemorrhage, rather than antiepileptic efficacy. For MTS, there is no consensus on the optimal dosing of radiation, and longterm procedural outcomes remain largely unknown. One prospective randomized study found superior efficacy of a higher radiation dose (24 versus $20 \mathrm{~Gy}$ ), though the antiepileptic effects still remained modest, ${ }^{24}$ while another demonstrated ineffectiveness

Peripheral zone: A peripheral T1-hypointense/T2-hyperintense zone surrounding the central zone represents necrotizing edema. This zone is irreversibly damaged and is not viable. Histopathologically, there is an inflammatory reaction, edema, an empty appearance of nerve cell processes and astrocytic foot processes, macrophages, and lymphocytes; this zone liquefies within few months. ${ }^{23}$

Outer rim of the peripheral zone: T1-hypointense/hyperintense and T2-hypointense ring bordering the peripheral zone is considered to represent damaged blood vessels with blood-brain barrier disruption and granulation tissue. The ring persistently enhances with gadolinium on postcontrast MR images and delineates the border of nonviable tissue. It is considered to represent deoxyhemoglobin initially, which evolves into hemosiderin deposition. $^{22}$

Vasogenic edema: T1-hypointense and T2-hyperintense regions of reversible vasogenic edema surround the hemosiderin/enhancing ring. This area appears days to weeks after the procedure, initially expands, and gradually shrinks after 2-9 weeks, with no overt residual tissue damage.

The ablation region (central and peripheral zones) initially increases during the first 2 weeks and then shrinks during the next 6 months. Enhancement may extend along the track of the laser catheter as well as in the peripheral rim. In the subacute period, the central zone $\mathrm{T} 1$ hyperintensity decreases, while the peripheral T1 hypointensity increases, leading to a more uniform appearance. The diameter of the enhancing rim decreases exponentially after mild initial increase, with a half-life of 93 days, but a focal area of enhancement can be seen for years. ${ }^{22}$ As stated above, vasogenic edema generally dissipates within 1-2 months. However, this idealized pattern may vary, particularly in the center of the lesion, as due to resultant radiation necrosis necessitating resective surgery. ${ }^{25}$ Imaging characteristics of the radiation-related parenchymal changes parallel typical brain radiation exposure with initial white matter signal alteration followed by edema and then radiation necrosis, typified by lace-like, irregular peripheral enhancement and central nonenhancing necrosis. Solid nodular enhancement may be seen with lesions of $<2 \mathrm{~cm} .{ }^{26}$ Cyst formation may occur in the mid- to long-term. Eventually, parenchymal involution, gliosis, and encephalomalacia occur months to years after treatment. Optimal imaging follow-up for irradiated MTS depends on the evolution of clinical symptoms; worsening focal seizures may precede edema and mass effect. ${ }^{24}$

\section{Neuromodulation}

As opposed to pharmacotherapy, which targets the entire brain, or surgical treatment, which consists of resection, disconnection, or ablation of certain anatomic regions, neuromodulation uses electrical stimulation to target specific regions of the brain to disrupt and modulate epileptic activity or networks. Currently, there are 3 main types of microinvasive neuromodulation methods that have FDA approval for the treatment of medically refractory epilepsy: vagus nerve stimulation (VNS), deep brain stimulation (DBS), and RNS. Other neuromodulation techniques such as transcranial magnetization, transcranial electrical stimulation, and focused sonography are under investigation. ${ }^{27}$ Familiarity with neuromodulation techniques, associated hardware, expected procedural outcomes, and their temporal evolution may prevent imaging pitfalls. While the effectiveness of seizure control by current neuromodulation devices is lower than resective surgery, these devices have a specified role in the treatment of intractable epilepsy (Fig 1). Implanted intracranial and extracranial 


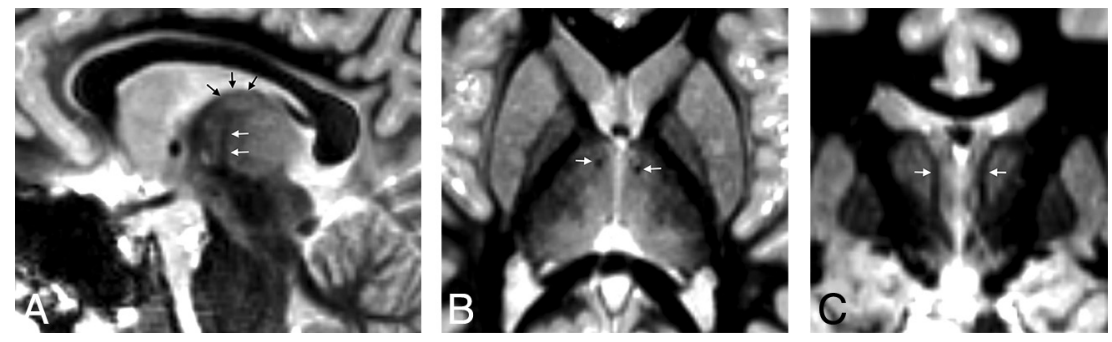

FIG 7. FGATIR, ANT localization. An 11-year-old child needing DBS because of bilateral poorly localized focal epilepsy despite left hippocampal sclerosis on MR imaging. ANT (black arrows) can be localized superior to the mammillothalamic track (white arrows), delineated on FGATIR parasagittal $(A)$, axial $(B)$, and coronal $(C)$ images. The FGATIR sequence nulls white matter in this track.

components should be compared with baseline postprocedural scans, particularly for location verification, and device migration or failure. The course of leads and extensions should be scrutinized for any structural signal change or fluid collection, particularly in case of suspected infections.

Vagus Nerve Stimulation. VNS was the first neuromodulation device used for intractable epilepsy, with FDA approval in 1997 as an adjunctive treatment for pharmacoresistant focal epilepsy. This technique is often used in patients with unidentifiable seizure foci or with multiple potential epileptogenic foci not amenable to interventional treatment. VNS is an extracranial procedure, with the infraclavicular subcutaneous implantation of a commercially available programmable pulse-generator device. Lead wires are attached to (and surround) the midcervical vagus nerve through a second neck incision via a subcutaneous tunnel (Online Supplemental Data). The generator runs continuously, but its activity can be controlled by the patient or programmer. VNS is placed on the left side to prevent damage to the sinoatrial node, which is innervated by the right vagus nerve. Patients with an implanted VNS device can undergo MR imaging using a transmit-receive head coil without a limit of active scanning time. ${ }^{28}$ For receive-only coils, specific conditions are applied for newer models, such as specific absorption rate restriction or a time limit for "safe active scanning," as little as 15 minutes, depending on the type and brand of the specific devices (VNS and/or DBS). On imaging, the position of the device, including wires, should be investigated. A potential complication of VNS is temporary or permanent left vocal cord paralysis. Other local or device-related complications are akin to those of neuromodulation systems in general, which are discussed in the DBS section below.

Responsive Neurostimulation. RNS has been an approved treatment for focal intractable epilepsy since 2013. It is indicated for a localized epileptogenic focus, in which there is a significant risk of a neurologic deficit associated with resection or ablation or for patients unwilling to undergo invasive therapies. As opposed to VNS and DBS, RNS has both recording and stimulation capability, with feedback similar to that of a cardiac pacemaker or defibrillator. Once abnormal electrocorticographic activities are detected, the RNS device (NeuroPace Inc., Mountain-view, CA, USA) will deliver electrical current pulses to disrupt and abort the ictal activity. ${ }^{29}$ RNS is a closed-loop system, which means that it delivers electrical impulses only after the targeted epileptiform activity is detected. A programmable neurostimulator device is implanted in the skull and connected to 2 subdural strips and/or depth electrodes (with 4 electrodes each) that are placed at or near a previously determined epileptogenic zone (Online Supplemental Data). Skull xrays are helpful in demonstrating the position of the device. CT is typically used for determination of intracranial device position as well as for potential surgical complications, but severe beam and streak artifacts limit evaluation. Angle cuts or dual-energy CT may provide further information in challenging cases. In March 2020, the FDA approved a commercial RNS device for MR imaging compatibility under appropriate conditions.

Deep Brain Stimulation. The discovery that damage to the anterior thalamic nucleus (ANT) in Rhesus monkeys decreased epileptogenic activity, likely by increasing the threshold of cortical epileptic discharges, led to the possibility of a new treatment technique, DBS. ${ }^{30}$ The ANT is part of the circuit of Papez, which relays information among the hippocampus, mamillary bodies, cingulum, and fornices. ${ }^{31}$ The Stimulation of the Anterior Nucleus of the Thalamus for Epilepsy (SANTE) trial and others demonstrated the safety and efficacy of ANT stimulation in pharmacoresistant focal epilepsy with reduction in seizures with variable results. ${ }^{32-34}$ The FDA recently approved commercially available MR imaging-conditional DBS systems for epilepsy treatment, which allow preprogramed intermittent stimulation of bilateral ANTs. Apart from the ANT, neurostimulation studies were also performed in the centromedian thalamus, subthalamic nucleus, caudate nucleus, hippocampus, hypothalamus, cortex, and cerebellum, which resulted in variable effects on seizures. For example, DBS targeting the centromedian thalamic nuclei has been shown to be effective in generalized epilepsy $^{35}$ due to circuitry connections to the medial frontal and cingulate gyri. ${ }^{36}$ Further understanding of epilepsy as a network disorder and new large-scale studies will likely broaden the clinical landscape of neuromodulation.

Although variations may occur in individual approaches to ANT stimulation across different institutions, the placement of electrodes is commonly performed in a transventricular fashion, which is connected to a subcutaneous generator (Online Supplemental Data). Electrode placement uses preoperative coregistered highresolution MR imaging and CT scans for frame-based stereotactic neuronavigation, with or without robotic assistance, and electrodes directed toward the anterior superior portion of the ANT. Before the report of Grewal et al, ${ }^{37}$ in 2018, describing direct targeting of the ANT, most surgeons used indirect targeting methods (eg, based on an atlas coregistered to the patient's anterior/posterior commissure line), which introduced some variability in electrode placement (including 10\% being placed outside the ANT). Grewal et al used 


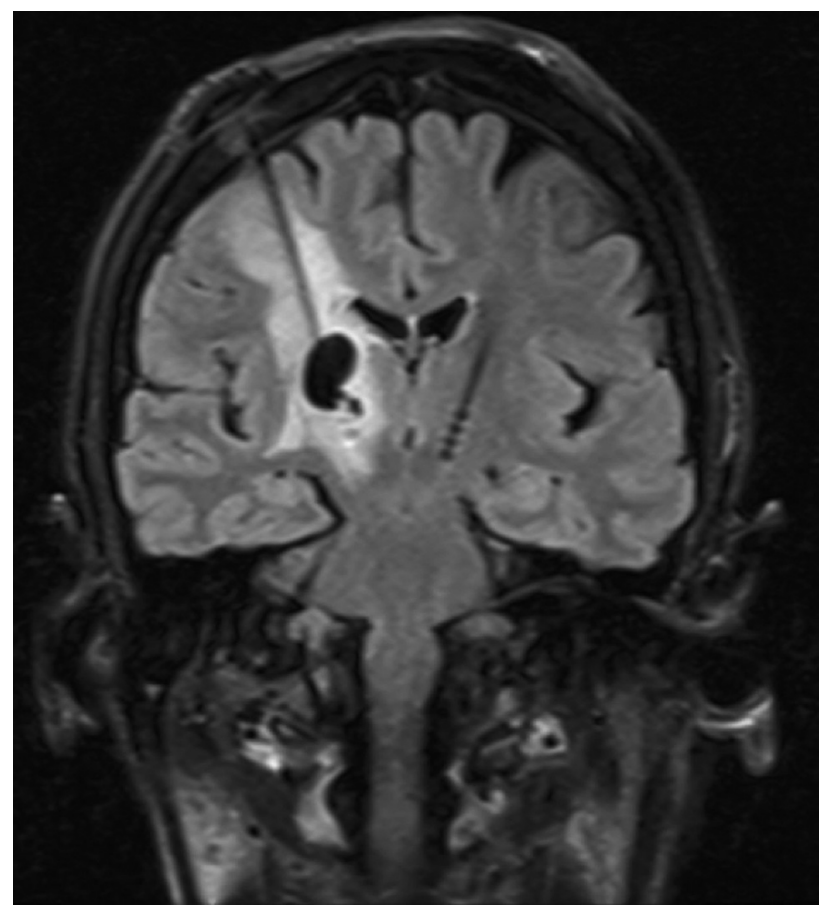

FIG 8. DBS complications in a patient without epilepsy. Left foot weakness and dysarthria occurred 3 months after bilateral DBS for refractory essential tremor. Coronal T2 FLAIR image demonstrates a nonhemorrhagic cyst with vasogenic edema surrounding the right electrode and no restricted diffusion or enhancement to suggest infection. This rare inflammatory DBS electrode complication was treated with steroids, resulting in regression of the cyst and vasogenic edema 7 weeks later (not shown here).

an 8-minute 0.8 -mm isotropic inversion recovery sequence (known as fast gray matter acquisition $\mathrm{T} 1$ inversion recovery or fast gray matter acquisition [FGATIR]), inorder to null the white matter to delineate the mammillothalamic track, in turn, allowing direct localization of the anterior nucleus of the thalamus situated superiorly (Fig 7). The mammillothalamic track is easily delineated on parasagittal FGATIR images, as opposed to 3D T1 gradient-echo imaging, which does not have sufficient contrast. MR or CT angiography can aid in the determination of vascular anatomy, for a safe trajectory.

The clinical effectiveness of DBS is primarily determined by the precision of the electrode implantation within the ANT and the burden of adverse effects. A long-term follow-up report from the SANTE trial demonstrated a procedural infectious complication rate of $12.7 \%$; however, none were intracranial. ${ }^{32}$ The neurostimulator migration rate was $5.5 \%$. Most other complications were neuropsychiatric disturbances. Delayed complications of DBS systems also include skin granuloma, bowstringing, extension lead failure, hardware migration, and perilead cyst formation and brain edema (Fig 8).

\section{CONCLUSIONS}

Pharmacoresistant epilepsy has a wide range of surgical interventional treatment options from microinvasive neuromodulation techniques to the functional hemispherectomy, which drastically alters cranial anatomy. As treatment options constantly evolve, practitioners need to be cognizant of unique imaging findings and potential short- and long-term complications associated with these interventions.

\section{ACKNOWLEDGMENT}

The authors thank the MR imaging safety officer and lead MR imaging technologist Ms Marie Hausner for her helpful discussions.

Disclosures: Jason Gerrard-RELATED: Consulting Fee or Honorarium: Medtronic, Longeviti Neuro Solutions, Comments: consultant for Medtronic; consultant for Longeviti Neuro Solutions; UNRELATED: consultancy for Boston Scientific. Richard Bronen—UNRELATED: Other: Pfizer, Comments: consultancy regarding brain imaging in patients with epilepsy, but unrelated to imaging after surgery or neuromodulation.

\section{REFERENCES}

1. Zentner J. Surgical Treatment of Epilepsies. In: Advances in Epilepsy Surgery and Radiosurgery. Springer, Vienna; 2002:27-35

2. Spencer DD, Gerrard JL, Zaveri HP. The roles of surgery and technology in understanding focal epilepsy and its comorbidities. Lancet Neurol 2018;17:373-82 CrossRef Medline

3. Spencer SS. Neural networks in human epilepsy: evidence of and implications for treatment. Epilepsia 2002;43:219-27 CrossRef Medline

4. Davis LM, Spencer DD, Spencer SS, et al. MR imaging of implanted depth and subdural electrodes: is it safe? Epilepsy Res 1999;35:95-98 CrossRef Medline

5. Merriam MA, Bronen RA, Spencer DD, et al. MR findings after depth electrode implantation for medically refractory epilepsy. AJNR Am J Neuroradiol 1993;14:1343-46 Medline

6. Nguyen HS, Doan N, Gelsomino M, et al. Dilemmas surrounding the diagnosis of deep brain stimulation electrode infection without associated wound complications: a series of two cases. Surg Neurol Int 2016;7:121-24 CrossRef Medline

7. Wiebe S, Blume WT, Girvin JP, et al. A randomized, controlled trial of surgery for temporal-lobe epilepsy. N Engl J Med 2001;345:31118 CrossRef Medline

8. Mansouri A. Surgery for drug-resistant epilepsy in children. $N$ Engl J Med 2018;378:399 CrossRef Medline

9. Noe K, Sulc V, Wong-Kisiel L, et al. Long-term outcomes after nonlesional extratemporal lobe epilepsy surgery. JAMA Neurol 2013;70:1003-08 CrossRef Medline

10. Schramm J, Clusmann H. The surgery of epilepsy. Neurosurgery 2008;62(Suppl 2):463-81; discussion 481 CrossRef Medline

11. Brotis AG, Giannis T, Kapsalaki E, et al. Complications after anterior temporal lobectomy for medically intractable epilepsy: a systematic review and meta-analysis. Stereotact Funct Neurosurg 2019;97:69-82 CrossRef Medline

12. Sato N, Bronen RA, Sze G, et al. Postoperative changes in the brain: MR imaging findings in patients without neoplasms. Radiology 1997;204:839-46 CrossRef Medline

13. Oztürk A, Oğuz KK, Akalan N, et al. Evaluation of parenchymal changes at the operation site with early postoperative brain diffusion-weighted magnetic resonance imaging. Diagn Interv Radiol 2006;12:115-20 Medline

14. Saluja S, Sato N, Kawamura Y, et al. Choroid plexus changes after temporal lobectomy. AJNR Am J Neuroradiol 2000;21:1650-53 Medline

15. Delev D, Oehl B, Steinhoff BJ, et al. Surgical treatment of extratemporal epilepsy: results and prognostic factors. Neurosurgery 2019;84:242-52 CrossRef Medline

16. Graham D, Tisdall MM, Gill D. Corpus callosotomy outcomes in pediatric patients: a systematic review. Epilepsia 2016;57:1053-68 CrossRef Medline 
17. Choudhri AF, Whitehead MT, McGregor AL, et al. Diffusion tensor imaging to evaluate commissural disconnection after corpus callosotomy. Neuroradiology 2013;55:1397-1403 CrossRef Medline

18. Morrell F, Whisler WW, Bleck TP. Multiple subpial transection: a new approach to the surgical treatment of focal epilepsy. J Neurosurg 1989;70:231-39 CrossRef Medline

19. Finet $P$, Grandin $C$, Vaz G, et al. Multiple subpial transections and magnetic resonance imaging. Neurochirurgie 2017;63:449-52 CrossRef Medline

20. Smith MC. Multiple subpial transection in patients with extratemporal epilepsy. Epilepsia 1998;39:S81-89 CrossRef Medline

21. Wu C, Jermakowicz WJ, Chakravorti S, et al. Effects of surgical targeting in laser interstitial thermal therapy for mesial temporal lobe epilepsy: a multicenter study of 234 patients. Epilepsia 2019;60:1171-83 CrossRef Medline

22. Schwabe B, Kahn T, Harth T, et al. Laser-induced thermal lesions in the human brain: short- and long-term appearance on MRI. $J$ Comput Assist Tomogr 1997;21:818-25 CrossRef Medline

23. Schober R, Bettag M, Sabel M, et al. Fine structure of zonal changes in experimental Nd:YAG laser-induced interstitial hyperthermia. Lasers Surg Med 1993;13:234-41 CrossRef Medline

24. Barbaro NM, Quigg M, Broshek DK, et al. A multicenter, prospective pilot study of gamma knife radiosurgery for mesial temporal lobe epilepsy: seizure response, adverse events, and verbal memory. Ann Neurol 2009;65:167-75 CrossRef Medline

25. Chen N, Du SQ, Yan N, et al. Delayed complications after Gamma Knife surgery for intractable epilepsy. J Clin Neurosci 2014;21:152528 CrossRef Medline

26. Wang YX, King AD, Zhou $\mathrm{H}$, et al. Evolution of radiation-induced brain injury: MR imaging-based study. Radiology 2010;254:210-18 CrossRef Medline

27. Stern JM, Spivak NM, Becerra SA, et al. Safety of focused ultrasound neuromodulation in humans with temporal lobe epilepsy. Brain Stimulation 2021;14:1022-31 CrossRef
28. de Jonge JC, Melis GI, Gebbink TA, et al. Safety of a dedicated brain MRI protocol in patients with a vagus nerve stimulator. Epilepsia 2014;55:e112-15 CrossRef Medline

29. Bergey GK, Morrell MJ, Mizrahi EM, et al. Long-term treatment with responsive brain stimulation in adults with refractory partial seizures. Neurology 2015;84:810-17 CrossRef Medline

30. Bouwens van der Vlis TA, Schijns O, Schaper F, et al. Deep brain stimulation of the anterior nucleus of the thalamus for drug-resistant epilepsy. Neurosurg Rev 2019;42:287-96 CrossRef Medline

31. Dalkilic EB. Neurostimulation devices used in treatment of epilepsy. Curr Treat Options Neurol 2017;19:7 CrossRef Medline

32. Salanova V, Witt T, Worth R, et al. SANTE Study Group. Long-term efficacy and safety of thalamic stimulation for drug-resistant partial epilepsy. Neurology 2015;84:1017-25 CrossRef Medline

33. Lee KJ, Shon YM, Cho CB. Long-term outcome of anterior thalamic nucleus stimulation for intractable epilepsy. Stereotact Funct Neurosurg 2012;90:379-85 CrossRef Medline

34. Krishna V, King NK, Sammartino F, et al. Anterior nucleus deep brain stimulation for refractory epilepsy: insights into patterns of seizure control and efficacious target. Neurosurgery 2016;78:802-11 CrossRef Medline

35. Valentín A, García Navarrete E, Chelvarajah R, et al. Deep brain stimulation of the centromedian thalamic nucleus for the treatment of generalized and frontal epilepsies. Epilepsia 2013;54:182333 CrossRef Medline

36. Kim SH, Lim SC, Yang DW, et al. Thalamo-cortical network underlying deep brain stimulation of centromedian thalamic nuclei in intractable epilepsy: a multimodal imaging analysis. Neuropsychiatr Dis Treat 2017;13:2607-19 CrossRef Medline

37. Grewal SS, Middlebrooks EH, Kaufmann TJ, et al. Fast gray matter acquisition T1 inversion recovery MRI to delineate the mammillothalamic tract for preoperative direct targeting of the anterior nucleus of the thalamus for deep brain stimulation in epilepsy. Neurosurg Focus 2018;45:E6 CrossRef Medline 